\title{
Approach to refractory hypoglycemia in the setting of pancreatic mass and commonly seen pitfalls
}

\author{
Samantha Edwards BA, Genanew Bedanie MD, Drew Payne DO
}

\begin{abstract}
An 86-year-old male presented with severe hypoglycemia and encephalopathy. Hypoglycemia workup determined the cause to be endogenous insulin. A pancreatic mass increased clinical suspicion for insulinoma, a rare pancreatic neuroendocrine tumor. Surgery is curative; however, the patient in this case was placed on comfort measures and died. This case report examines the clinical guidelines for evaluation, diagnosis, and management of hypoglycemia in persons without diabetic mellitus.
\end{abstract}

Keywords: Hypoglycemia, testing, pancreatic mass

\section{INTRODUCTION}

Pancreatic neuroendocrine tumors (NETs) are rare neoplasms that form in the endocrine tissue of the pancreas. The majority of these tumors are non-functioning, yet functioning NETs can secrete hormones, such as insulin, gastrin, vasoactive intestinal peptide (VIP), and glucagon, leading to multiple signs and symptoms. The diagnosis of an insulinoma can be challenging and confused with other causes of hypoglycemia. The pathophysiology, workup, diagnosis, and management of hypoglycemia in diabetes are distinct from the evaluation in a patient without diabetes. ${ }^{1}$ A review of the evaluation and workup for hypoglycemic disorders in adults will contribute to timely and proper diagnoses, including insulinomas.

\section{CASE}

An 86-year-old man was presented to the emergency department via the emergency medical services (EMS) after being found down and unresponsive at his

Corresponding author: Drew Payne Contact Information: Drew.payne@ttuhsc.edu DOI: 10.12746/swrccc.v8i33.635 geriatric care center. EMS found the capillary blood glucose at the scene to be $26 \mathrm{mg} / \mathrm{dL}(65-115 \mathrm{mg} / \mathrm{dL})$ and administered $50 \mathrm{~mL}$ of glucose $(50 \mathrm{~g} / \mathrm{dL})$. Physical examination on presentation to the emergency department showed an unresponsive patient with a blood pressure of $142 / 92 \mathrm{mmHg}$, a respiratory rate of 20 breaths per minute, and a heart rate of 55 beats per minute. The patient was intubated for airway protection and given dextrose solutions to increase his blood sugar. The patient had been discharged the day before after a 14-day hospitalization for an upper gastrointestinal bleed (UGIB) from a duodenal ulcer, severe anemia, complicated by pneumonia, and acute kidney injury on stage III chronic kidney disease. Relevant labs upon admission revealed a plasma glucose of $121 \mathrm{mg} / \mathrm{dL}$ and a hemoglobin A1C of $4.7 \%$ (4.0-6.0\%). Over the next few days of hospitalization, the patient continued to be hypoglycemic with blood sugars in the 40 s and required a continuous infusion of fluids with dextrose and remained encephalopathic.

\section{INVESTIGATION}

On the prior admission, ultrasound and computed tomography (CT) scan of the abdomen detected a $2 \mathrm{~cm} \times$ $2 \mathrm{~cm}$ mass in the uncinate of the pancreas (Figure 1) and a right hepatic lobe cyst (Figure 2). Outpatient workup of the mass had been recommended. During 


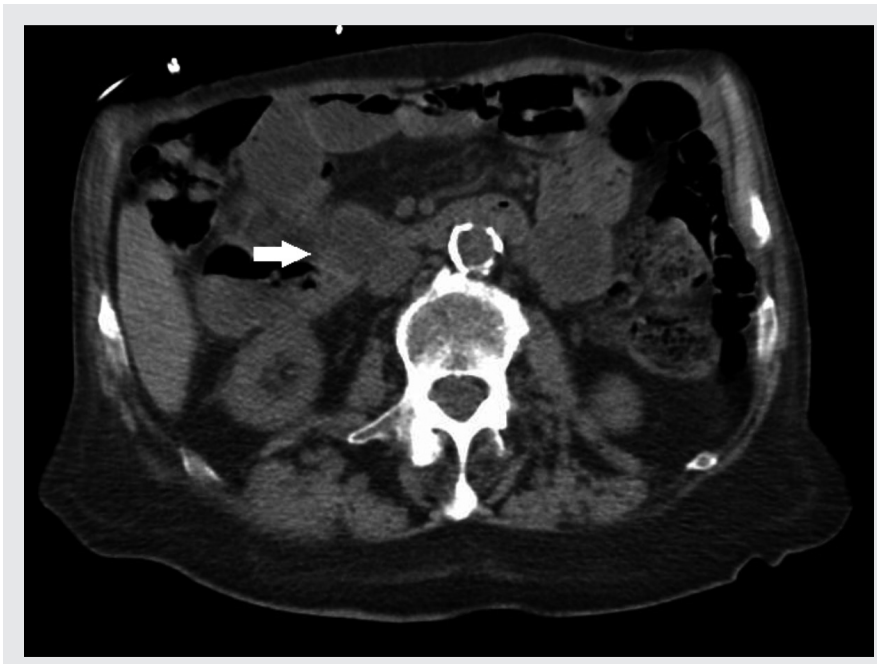

Figure 1. CT with pancreatic mass located in the uncinate head of the pancreas.

this admission, endocrinology service was consulted, and a diagnostic 72-hour fast was initiated to determine the etiology of hypoglycemia. Blood glucose was monitored every hour until it fell below $55 \mathrm{mg} / \mathrm{dL}$. Symptomatic hypoglycemia occurred after four hours of fasting and the following blood tests were drawn: plasma glucose $43 \mathrm{mg} / \mathrm{dL}$ insulin $15.4 \mathrm{U} / \mathrm{mL}(2.6-24.9 \mathrm{U} /$ $\mathrm{mL})$, C-peptide $21 \mathrm{ng} / \mathrm{mL}(1.1-4.4 \mathrm{ng} / \mathrm{mL})$, proinsulin

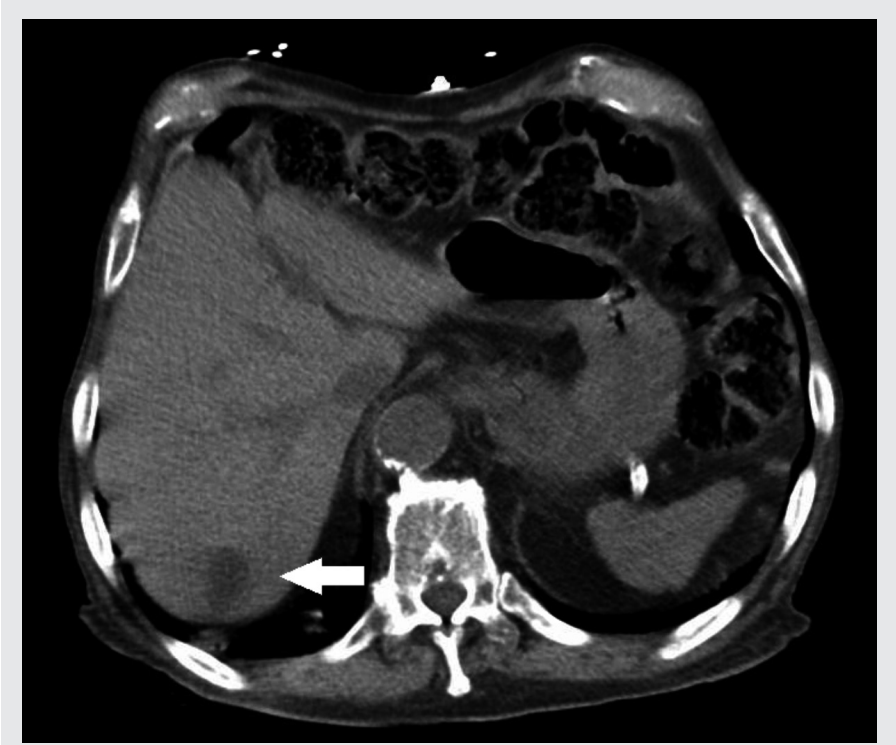

Figure 2. CT demonstrating cyst in right lobe of liver.
Table 1. Clinical diagnostic values for insulinoma

\begin{tabular}{|l|l|}
\hline Chlorpropramide & None Detected \\
\hline Tolazamide & None Detected \\
\hline Pioglitazone & None Detected \\
\hline Glimepiride & None Detected \\
\hline Repaglinide & None Detected \\
\hline Rosiglitazone & None Detected \\
\hline Tolbutamide & None Detected \\
\hline Glipizide & None Detected \\
\hline Glyburide & None Detected \\
\hline Nateglinide & None Detected \\
\hline
\end{tabular}

$121.3 \mathrm{pmol} / \mathrm{L}$ (18 pmol/L), and B-hydroxybutyrate $0.05 \mathrm{mmol} / \mathrm{L}(0.28 \mathrm{mmol} / \mathrm{L})$ (Table 2$)$. The patient was given $1 \mathrm{mg}$ IV glucagon, the fast was terminated, and the patient was treated with IV dextrose. Blood sugar was monitored per protocol; an increase in serum blood sugar from $43 \mathrm{mg} / \mathrm{dL}$ to $74 \mathrm{mg} / \mathrm{dL} 10$ minutes after intravenous administration of glucagon was measured. In addition, no oral hypoglycemic agents or insulin antibodies were detected (Table 1 and 2). The next step would have been an MRI to better visualize the pancreatic mass followed by a biopsy. However, the family decided to pursue comfort measures, so these steps were not performed.

\section{DifFERENTIAL DIAGNOSIS}

A correct differential diagnosis is crucial to avoid invasive procedures, like surgery, and to secure proper treatment for the etiology of the hypoglycemia. Individuals experiencing hypoglycemia in the absence of diabetes mellitus should be categorized into "ill" or "seemingly well" individuals. Drugs, critical illness, hormone deficiency, and non-islet cell tumors should be included in the differential diagnosis for ill patients. For apparently healthy patients, endogenous hyperinsulinemia, as well as accidental, surreptitious, or malicious hypoglycemia are probably causes. ${ }^{2}$

It is difficult to classify the patient presented in this case into ill or seemingly well. While he was recently hospitalized for UGIB and had chronic kidney disease, he lacked other major comorbidities. Nonetheless, 
Table 2. Screen for circulating oral hypoglycemic agents

\begin{tabular}{|l|c|c|c|c|c|c|c|c|}
\hline & $\begin{array}{c}\text { Plasma } \\
\text { Glucose } \\
(\mathbf{m g} / \mathbf{d L})\end{array}$ & $\begin{array}{c}\text { Insulin } \\
(\mathbf{U} / \mathbf{m L})\end{array}$ & $\begin{array}{c}\text { C-peptide } \\
\mathbf{( n g / L )}\end{array}$ & $\begin{array}{c}\text { Proinsulin } \\
\text { (pmol/L) }\end{array}$ & $\begin{array}{c}\text { Glucose } \\
\text { B-hydroxy } \\
\text { butyrate } \\
\mathbf{( m m o l / L )}\end{array}$ & $\begin{array}{c}\text { increase after } \\
\text { glucagon } \\
\text { (mg/dL) }\end{array}$ & $\begin{array}{c}\text { Insulin } \\
\text { Auto Ab }\end{array}$ & $\begin{array}{c}\text { Signs, } \\
\text { symptoms } \\
\text { or both }\end{array}$ \\
\hline Normal fasting & $<55$ & $<3$ & $<0.6$ & $<5$ & $>2.7$ & $<25$ & Neg & No \\
\hline $\begin{array}{l}\text { Insulinoma, NIPHS, } \\
\text { PGBH }\end{array}$ & $<55$ & $\geq 3$ & $\geq 0.6$ & $\geq 5$ & $\leq 2.7$ & $>25$ & Neg & Yes \\
\hline Patient Values & 43 & 15.4 & 21 & 121.3 & 0.05 & 28 & Neg & Yes \\
\hline
\end{tabular}

NB: Circulating oral hypoglycemic agents should be negative; Neg-negative; Ab-antibody;

NIPHS - Noninsulinoma pancreatogenous hypoglycemia syndrome; PGBH - Post-Gastric Bypass Surgery

Hypoglycemia; Modified from: Evaluation and Management of Adult Hypoglycemic Disorders: An Endocrine Society Clinical Practice Guidelines ${ }^{3}$

his lab values indicating elevated insulin levels in the presence of low plasma glucose narrow the differential diagnosis. The possibilities for hyperinsulinemic hypoglycemia in the absence of diabetes mellitus consists of oral hypoglycemic agents, exogenous insulin, endogenous insulin, and antibodies to native insulin.

The absence of circulating oral hypoglycemic agents and antibodies against insulin negated these diagnoses. The diagnostic feature of endogenous hyperinsulinism is the failure of insulin levels to fall while hypoglycemic plasma glucose levels occur. This is evaluated by measuring at three critical diagnostic features: plasma insulin, plasma c-peptide, and plasma proinsulin. Elevated insulin, c-peptide, and proinsulin levels at the time of hypoglycemia invalidates exogenous insulin and suggests insulin-dependent hypoglycemia (Figure 3). This information, combined with a known pancreatic mass, increased clinical suspicion for an insulinoma rather than noninsulinoma pancreatogenous hypoglycemia syndrome..$^{2,3}$

\section{TREATMENT}

The gentleman in this case was not treated for the suspected insulinoma since he was placed on comfort measures. However, surgical resection is typically curative for benign insulinomas, with rates approaching $100 \% .{ }^{4}$ Restrictions exist for those with Multiple Endocrine Neoplasia 1 (MEN1) and metastatic insulinomas. The hypoglycemic symptoms can be managed medically and by lifestyle modifications if surgery is not possible. Frequent, small meals can help control symptoms. In addition, diazoxide can be successful at managing symptoms in $50 \%$ to $60 \%$ of patients. Long acting somatostatin analogs can also be helpful in patients with insulinomas that are nonmetastatic, although the risk of worsening hypoglycemic symptoms does exist. ${ }^{2,4}$

\section{OUTCOME AND FOLLOW UP}

Due to the patient's rapid decline and encephalopathy, comfort measures were requested (hospital day \#7) and he died (hospital day \#9) due to hypoglycemia and severe sepsis secondary from aspiration pneumonia.

\section{Discussion}

Insulinoma is a rare pancreatic beta-cell tumor that has an incidence of 1 in 250,000 patient-years and is more common in women $(60 \%)$ than in men. ${ }^{1}$ Insulinomas occurs at all ages with a median age of diagnosis of 50 years, are mostly benign (90-95\%), solitary (93\%), confined to the pancreas (99\%), and small (avg. 1-2 cm). ${ }^{1}$ Insulinomas often remain undiagnosed and symptoms are attributed to psychiatric or neurologic disorders for several years. The long-term hyperinsulinemia, caused by excessive insulin secretion that does not respond to glucose concentrations, can lead to persistent hypoglycemia. Symptoms of an insulinoma are typically made worse when fasting and manifests as neuroglycopenic symptoms, such as confusion, altered consciousness, sweating, shakiness, tachycardia, anxiety, and the sensation of hunger. ${ }^{4}$ 


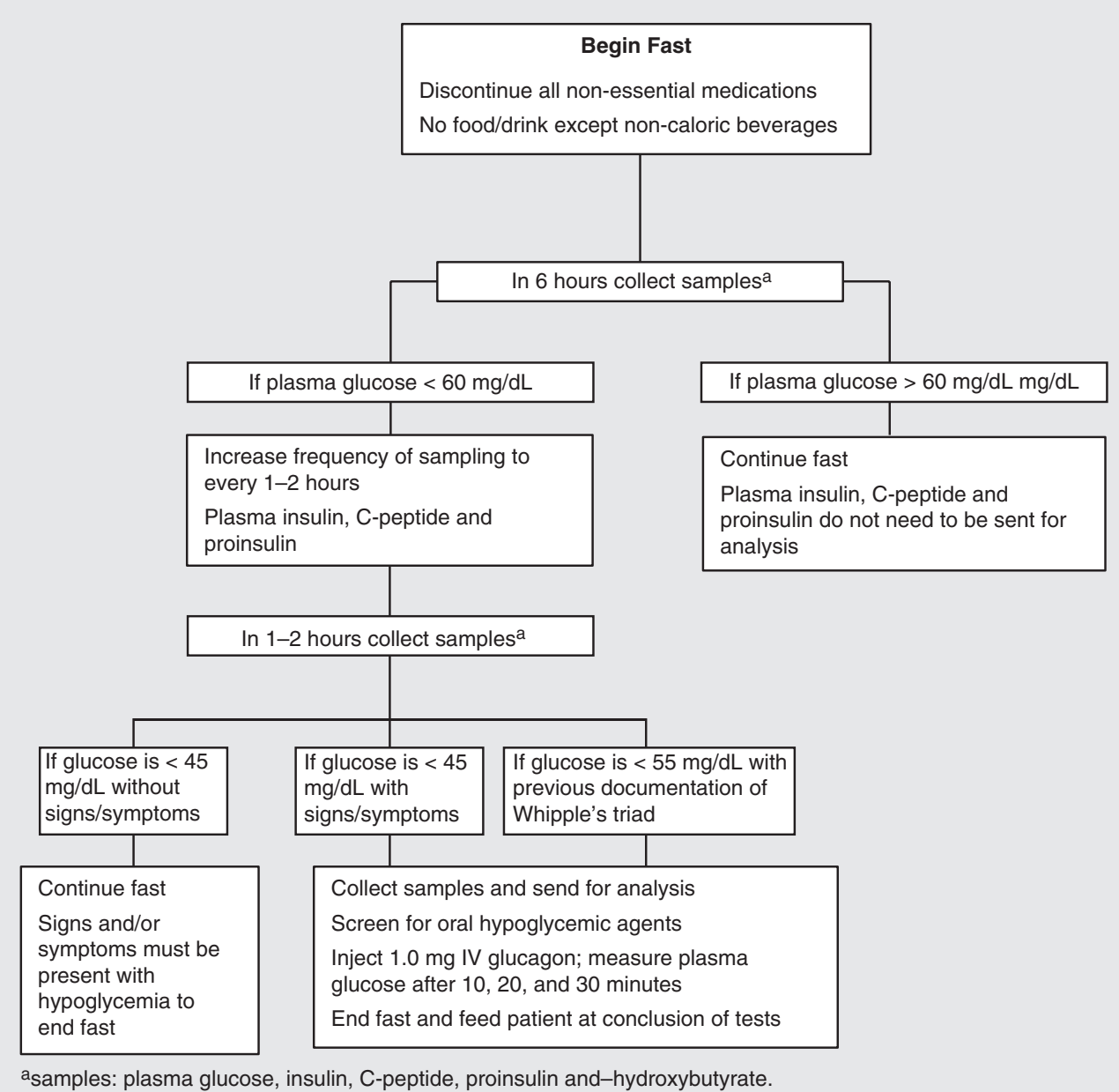

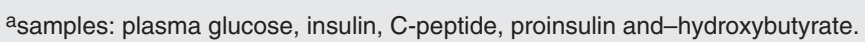

Figure 3. Protocol for 72-hour fast.

The hypoglycemia protocol utilized in this case is consistent with the Society of Endocrinology Practice Guidelines and is detailed here. Hypoglycemia workup was performed based on the demonstration of Whipple's triad, consisting of signs and symptoms of hypoglycemia, low plasma glucose concentration, and the resolution of symptoms upon administration of glucose. ${ }^{2}$ Once Whipple's triad is documented, further evaluation and workup are warranted. Testing should be performed during a spontaneous hypoglycemic episode with the presence of symptoms. If this is not possible, a 72 hour fast should be done for diagnosis of the etiology of the hypoglycemia and confirmation that the symptoms experienced are caused by the hypoglycemia (Table 1). ${ }^{4}$

The 72-hour fast should be done in a monitored setting and consists of measuring plasma glucose, insulin, insulin antibodies, C-peptide, proinsulin, and $\beta$-hydroxybutyrate concentrations, as well as a screen for oral hypoglycemic agents and the change in plasma glucose in response to intravenous injection of $1.0 \mathrm{mg}$ glucagon. Guidelines state that plasma glucose, insulin, proinsulin, $\beta$-hydroxybutyrate, and C-peptide should be collected every 6 hours until plasma glucose falls below $60 \mathrm{mg} / \mathrm{dL}$, at which point samples should be measured every 1-2 hours. Insulin, C-peptide, and proinsulin need to be sent for analysis only when the plasma glucose measures less than $60 \mathrm{mg} / \mathrm{dL}^{2}$ The fast is continued until the plasma glucose is less than $45 \mathrm{mg} / \mathrm{dL}$, and the patient is showing signs and/or symptoms of hypoglycemia. Alternatively, if Whipple's triad had been previously demonstrated, the fast can be terminated when the blood glucose reaches less than $55 \mathrm{mg} / \mathrm{dL}$ 
in the absence of current signs and/or symptoms. At this time proinsulin, beta-hydroxybutyrate, plasma glucose, insulin, C-peptide, and oral hypoglycemia agents should be measured, and the fast is terminated. ${ }^{2,3}$ It is important that laboratory measured plasma glucose be used to decide to end the fast, rather than a point of care glucose monitor. A glucagon tolerance test should be conducted in which $1.0 \mathrm{mg}$ of glucagon is administered and plasma glucose is measured 10, 20 and 30 minutes after administration. Further testing can include insulin antibodies, IGF-1/IGF-2, plasma cortisol, glucagon, or growth hormone depending on the suspected etiology. ${ }^{3}$

In the case presented here, the patient was on a constant dextrose drip and was still experiencing hypoglycemic episodes. Stopping the dextrose and initiating the fast was predicted to cause the glucose levels to drop significantly. For this reason, his capillary blood glucose was checked hourly. After four hours, the capillary blood glucose dropped to $46 \mathrm{mg} / \mathrm{dL}$, and plasma glucose was collected and measured at $43 \mathrm{mg} / \mathrm{dL}$, confirming that the fast should be terminated per the Journal of Clinical Endocrinology and Metabolism Guidelines. Patients with insulinomas often fall below the $55 \mathrm{mg} / \mathrm{dL}$ plasma glucose threshold well before 72 hours with two-thirds of patients ending the fast in 24 hours. $^{2,3}$ The laboratory values collected when the plasma glucose measured $46 \mathrm{mg} / \mathrm{dL}$ are consistent with the diagnostic value for an insulinoma (Table 3). While the insulin value of $15.4 \mathrm{U} / \mathrm{mL}$ falls within the normal range, it is abnormal in the presence of hypoglycemia. The presence of Whipple's triad, a pancreatic mass, inappropriately high insulin, proinsulin, and c-peptide, and the lack of oral hypoglycemic agents and circulating insulin antibodies support the diagnosis of an insulinoma.

\section{KEY POINTS}

1. Isolated hypoglycemia in the absence of clinical symptoms/Whipple triad does not define hypoglycemia.

2. While the differential diagnosis for hypoglycemia is broad, a thorough review and adherence to the guidelines for hypoglycemic workup is essential for an accurate and timely diagnosis.

3. Simultaneous measurement of blood sugar, insulin and C-peptide level during clinical symptoms of hypoglycemia is the only way to provide evidence for an inappropriate insulin secretion. Once inappropriate insulin secretion is confirmed, presence of sulfonylurea and anti-insulin antibodies should be ruled out. This can be followed by imaging to evaluate the pancreas for masses and possible insulinoma.

4. Insulinomas can have an indolent course and can be challenging to diagnose.

Article citation: Edwards S, Bedanie G, Payne D. Approach to refractory hypoglycemia in the setting of pancreatic mass and commonly seen pitfalls. The Southwest Respiratory and Critical Care Chronicles 2020;8(33):47-51

From: Department of Internal Medicine, Texas Tech University Health Sciences Center, Lubbock, Texas

Submitted: $12 / 8 / 2019$

Accepted: 12/20/2019

Reviewer: Marcella Rivas MD

Conflicts of interest: none

This work is licensed under a Creative Commons

Attribution-ShareAlike 4.0 International License.

\section{REFERENCES}

1. Benjamin IJ, Griggs RC, Wing EJ, et al. Andreoli and Carpenter's Cecil Essentials of Medicine. 9th edition. Philadelphia, PA: Elsevier/Saunders; 2016, p. 1190, xxv.

2. Cryer PE, Axelrod L, Grossman AB, et al. Evaluation and management of adult hypoglycemic disorders: An Endocrine Society Clinical Practice Guideline. J Clin Endocrinol Metab 2009;94(3):709-28.

3. Desimone ME, Weinstock RS. Non-Diabetic Hypoglycemia. In: De Groot LJ, Chrousos G, Dungan K, et al. Editors. Endotext. South Dartmouth (MA) 2000.

4. Kulke MH, Anthony LB, Bushnell DL, et al. NANETS treatment guidelines: well-differentiated neuroendocrine tumors of the stomach and pancreas. Pancreas 2010;39(6):735-52. 